\title{
Cues reduce direction uncertainty and enhance motion detection
}

\author{
KARLENE BALL and ROBERT SEKULER \\ Cresap Neuroscience Laboratory, Northwestern University, Evanston, Illinois 60201
}

\begin{abstract}
Previous work has shown that detectability of motion is better when the observer knows ahead of time the direction of that motion ("certainty") than when he does not know the direction ("uncertainty"). We now report attempts to reduce this performance decrement associated with direction uncertainty. In these experiments, a briefly flashed, oriented line cued the observer to the direction of motion that might occur. When the cue appeared before the moving target, detectability increased; when the cue appeared after the moving target, performance dropped below that for no cue at all. In addition, we examined the effect of cue reliability, varying the relation between cue orientation and actual direction of target motion. The more accurate the cue is, the larger the performance increment. When the cue indicated a direction more than $90 \mathrm{deg}$ from the actual target direction, performance was worse than when there was no cue. Results are discussed with regard to the feasibility of reducing uncertainty in real-world situations.
\end{abstract}

Observers who know the precise characteristics of a target will detect it more easily than will observers who are uncertain about the target's characteristics. The loss in detectability accompanying stimulus uncertainty has been studied in the domains of wavelength (Greenhouse \& Cohn, 1978), spatial position (Cohn \& Lasley, 1974), and target motion (Sekuler \& Ball, 1977), among others. We have been particularly interested in the decrease in detectability of a moving target when the observer is uncertain about its direction of motion.

There are several reasons for this continuing interest. First, since uncertainty about direction of motion produces larger performance decrements than do other sources of uncertainty, it provides a convenient arena within which to study uncertainty effects generally. Second, properly exploited, the consequences of direction uncertainty can illuminate the properties of visual mechanisms responsible for the perception of target motion (Ball \& Sekuler, 1980). Third, since most of our everyday perception of motion occurs under conditions of direction uncertainty, we must understand how uncertainty operates in order to extrapolate from laboratory studies to the circumstances most commonly encountered outside the laboratory. Fourth, work with direction uncertainty allows us to examine a hybrid process partaking of both cognitive processes (since it resembles attention) as well as sensory processes (since it is sense dominated). This hybrid character of direction uncertainty is particularly attractive to those who are interested in cognitive influences over perception.

This research was supported by Grant AF-79-0064 from the U.S. Air Force. We thank Randolph Blake and Dave Westendorf for helpful suggestions on an earlier version of this paper.
To all these reasons for examining motion uncertainty, the present study adds yet another. In many situations outside the laboratory, an observer's uncertainty about the characteristics of potential targets significantly limits the observer's performance. Among the situations we have in mind are driving cars and other vehicles, monitoring radar and similar displays, and piloting airplanes. In all these tasks, the observer's performance could be significantly enhanced if uncertainty could be reduced.

One way in which this could be accomplished would be with a real-time sensory aid working in parallel with the human observer. In the extreme, if the device's information were as detailed and accurate as that of an unhurried human observer, the human observer would be redundant. More generally, the information provided by this sensory aid could help the observer narrow the range over which he had to look for possible targets. If the hardware provided the observer with rapid but crude supplemental information, targets could be rendered more visible and latency of response to the targets reduced.

Since such devices could be developed with existing technology, we wished to determine how much help they might actually be to a human observer. To answer this question, we examined the ability of direction cues to reduce the detectability loss that direction uncertainty usually produces. In addition to the practical applications of such information, we were also interested in enhancing our understanding of the properties of visual mechanisms underlying our ability to perceive motion.

Several models have been proposed to account for the effects of uncertainty in perceptual tasks. These have been described in detail elsewhere (Ball \& Sekuler, 1980; Johnson \& Hafter, 1980). The sort of models 
that appear to be most adequate for motion perception postulate that the observer is sensitive only to a limited range of directions at any one moment. Although the observer is capable of changing the specific range of directions being monitored, this change is far from instantaneous. In fact, it requires enough time for it to be impossible to monitor two widely separated directions during a single brief observation interval (Ball \& Sekuler, 1980).

In previous work on direction uncertainty (Ball \& Sekuler, 1981), we showed that an observer has control over the range of directions he monitors. In particular, as the ensemble of possible stimuli changes from one block of trials to another, there are corresponding changes in the band of directions that the observer monitors. In order to make maximum use of supplementary prompts provided by a sensory aid, however, a human observer would have to alter the direction he was monitoring in a fraction of a second. Since, in our previous work, the stimulus ensemble changed only between blocks of trials, we do not know how rapidly an observer can actually alter his observation band.

One aim of the present study was to determine whether rapid and flexible deployment of sensory resources was actually possible. We simulated this realworld situation in our laboratory by allowing observers varying amounts of time to alter the direction being monitored following the presentation of a cue. In the three experiments reported here, we used a briefly flashed line as a prompt, its orientation signifying the direction of motion that the observer would have to detect.

\section{EXPERIMENT 1}

In the first experiment, the prompt line was presented at various times relative to the moving target. Detectability aided by this cue was compared with detectability in two control conditions: (1) with the cue omitted entirely, and (2) with no direction uncertainty whatever. To determine the source of potential changes in response to the cue, we used a psychophysical method that would give independent estimates of sensitivity and criterion.

\section{Method}

Obuervers. Three paid observers participated in this experiment. All were experienced psychophysical observers, and all had uncorrected $20 / 20$ vision.

Apparatus. Stimuli were isotropic random-dot patterns presented under computer control on a cathode ray tube display (CRT). The target to be detected consisted of approximately 400 dots that could appear within an 8-deg-diam circular aperture in a mask over the CRT. Patterns were presented against a constant veiling luminance of $1.7 \mathrm{~cd} / \mathrm{m}^{2}$. The observer was seated in a darkened booth with his head supported in a chinrest $57 \mathrm{~cm}$ from the display. Viewing was binocular, and the observer was required to fixate a small dot in the center of the screen. Dots could move upward, leftward, rightward, or downward, but always at
$4.0 \mathrm{deg} / \mathrm{sec}$, the speed used in our earlier studies of uncertainty (Ball \& Sekuler, 1980).

Procedure. A session consisted of 80 trials. During each trial, a tone defined an interval of $500 \mathrm{msec}$ duration (hereafter, the observation interval), during which moving dots might appear on the CRT. In fact, a moving pattern was presented on only one-half of the trials, chosen at random. On the remaining trials, the CRT was illuminated only by the veiling light during the observation interval.

A rating scale method was used to measure sensitivity and criterion (McNicol, 1972). Following each trial, the observer responded by pushing one of six response buttons, one corresponding to each category on the rating scale. Instructions defined the rating scale as follows: 1 , positively was a moving pattern on the screen; 2, probably was a moving pattern on the screen; 3, possibly was a moving pattern on the screen; 4 , possibly was no moving pattern on the screen; 5, probably was no moving pattern on the screen; and 6, positively was no moving pattern on the screen. The observer was instructed to try to use all six response categories if at all possible and was told that a " 1 " or a " 6 " was no more a "right" answer than was a "2," "3," "4," or "5."

Detectability of the moving dots was measured in three conditions: (1) certainty, (2) uncertainty, and (3) cued uncertainty. During a session of 80 trials, the condition was unchanged.

In the certainty condition, the observer was informed prior to the session which direction would be presented and, to eliminate all uncertainty, was also shown the moving dot pattern. The same direction was presented throughout the block of 80 trials. Each trial consisted of a warning signal followed by a 500 -msec observation interval during which the movement could occur (probability of .5). The observer then gave his rating response by pushing one of six response buttons.

In the uncertainty condition, the dots could move in one of four possible directions. The direction was chosen randomly from the four possibilities (upward, downward, leftward, and rightward) under the constraint that over a session of $\mathbf{8 0}$ trials all four would occur equally often. The observer's task was to rate his certainty that moving dots had or had not been presented. No judgment about direction of movement was required.

In the cued uncertainty condition, trials were identical to those in the uncertainty condition except that, on each, the observer received a cue to the direction that might occur. The cue consisted of a line extending outward 5 deg from the center of the CRT. Dotted at its outer edge and pointing in the direction of possible stimulus movement, the cue was always presented for $50 \mathrm{msec}$. For those trials on which no moving dots would be presented, the orientation of the cue was randomly chosen from among the four alternative orientations, again under the constraint that all four would occur equally often in the 80-trial session.

To determine how rapidly observers could adjust, the cue was presented at various times either before or after the observation interval. In particular, the cue was presented $0,50,100,200,300$, 450 , or $700 \mathrm{msec}$ prior to the observation interval or $0,50,100$, $200,300,450$, or $700 \mathrm{msec}$ after the observation interval. For the conditions in which the cue preceded the observation interval, the delay time was defined as the interval between the offset of the 50 -msec cue and the onset of the observation interval. For those conditions in which the cue followed the observation interval, the delay time started at the offset of the observation interval and ended with the onset of the cue. As before, the observer used a set of six buttons to indicate his rating. Only one time condition was presented within each block of $\mathbf{8 0}$ trials, and each observer participated in each condition once. Order of condition presentation was determined randomly for each of the three observers.

Two preliminary sets of measurements preceded the actual experiment. The first determined the accuracy with which the orientation of the s0-msec cue could itself be perceived. Obviously, unless the cue were accurately perceived, it could not be of much help to the observer. To answer this question, we tested the accuracy of observers in naming the direction specified by the cue. 
On any trial, only the cue line was presented, its orientation chosen randomly from the 360 possibilities.

Following each $50-\mathrm{msec}$ flash of the cue line, the observer read its perceived orientation off a protractor that surrounded the CRT. We found that the mean absolute error in identification of the cue orientation was between 3 and 4 deg. No significant constant errors were observed, although accuracy of judging cues oriented on the horizontal and vertical axes was somewhat better than for those presented in oblique orientations. These measurements correspond closely to direction difference thresholds measured previously with the method of constant stimuli (Ball \& Sekuler, 1979). This finding, added to the finding of an "oblique effect" (Appelle, 1972), confirms that the cue was accurately perceived. This level of accuracy is particularly good given that the line was flashed for only $50 \mathrm{msec}$ (cf. Andrews, 1965). In addition, tests showed that a similar line did not alter the perceived direction of moving dots (Levinson \& Sekuler, 1980).

The second type of preliminary measurement identified a luminance for the moving dots that would produce the desired overall level of detectability. We needed a luminance that would produce greater-than-chance detection in the most difficult condition (uncertainty) but less than perfect detection in the easiest condition (certainty). To find the appropriate level for each observer, we ran alternating blocks of certainty and uncertainty trials at various luminances. Once the desired luminance had been identified, the same value was used for that observer throughout the experiment.

\section{Results}

To describe each observer's performance, we used two different measures, one related to sensitivity and the other related to the observer's criterion. Procedures for calculating these measures are given in McNicol (1972). P(A), a nonparametric measure of detectability, is the proportion of the area of the unit normal square that lies below the receiver operating characteristic. Larger values of $P(A)$ signify that the movement was more easily detected. $P(A)$ is not linearly related to sensitivity, $d^{\prime}$, but $\mathrm{zP}(\boldsymbol{A})$ is. As a result, we used z-transformed values of $P(A)$ to define sensitivity. The second measure derived from our data, B, indicates an observer's bias for or against saying that the stimulus had been presented. Higher $B$ values indicate a tendency to deny that moving dots had been presented.

Figure 1 shows the $\mathrm{zP}(\mathrm{A})$ values obtained for the three observers in each condition. The far right-hand points on the graph represent the uncertainty condition with no cue and the far left-hand points, the certainty condition. For conditions in which the cue preceded the observation interval, note that as the time interval between the cue and the observation interval increased, $\mathrm{zP}(\mathrm{A})$ also increased. For conditions in which the cue followed the observation interval, $\mathrm{zP}(\mathrm{A})$ was invariant as the time interval increased.

A one-way analysis of variance revealed that results from the three conditions of the experiment differed significantly $[F(15,30)=53.14, p<.01]$. A NewmanKeuls test showed that when the cue followed the observation interval, detection was not significantly different from detection with no cue at all $(p<.05)$. The same test revealed that with cues preceding the observation interval by either 450 or $700 \mathrm{msec}$, detec-

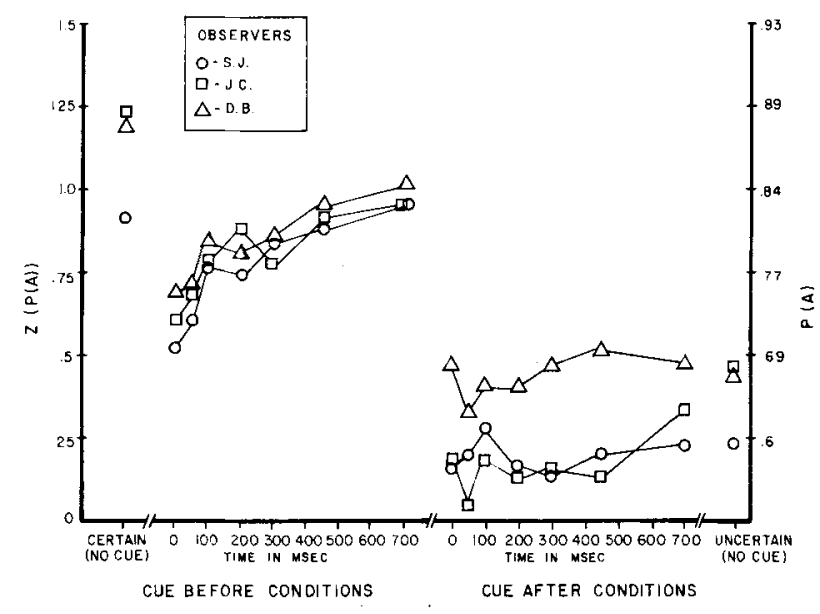

Figure 1. Sensitivity, $\mathrm{zP}(\mathrm{A})$, for each condition and observer in Experiment 1. Far left-hand points represent certainty with no cue. Far right-hand points represent uncertainty with no cue. Conditions in which the cue preceded the stimulus are plotted to the left as a function of time of arrival of the cue. The conditions in which the cue followed the stimulus are plotted on the right as a function of the time interval between observation and cue presentation.

tion was not significantly different from detection with complete certainty about direction.

$B$ values were calculated for each observer in each condition. A one-way analysis of variance did not reveal a significant effect of conditions $(p>.25)$, indicating that the observers' criterion for saying dots were presented was constant across all conditions. Since observers seemed to use the same criterion for reporting the presence of motion under all conditions of certainty or uncertainty, the changes in $\mathbf{z P}(\mathbf{A})$ we observed can be attributed to sensitivity changes without concern for possible covariation in criterion.

Finally, averages were computed across observers for the transformed $P(A)$ values. Lines were then fit separately to both the cue-after and cue-before conditions. The cue-after conditions were best described by a line in which $\mathrm{zP}(\mathrm{A})=.11 \mathrm{x}+.24$, where $\mathrm{x}$ is the interval in seconds. The small slope coefficient and the large percentage of variance unaccounted for by this equation ( $59 \%$ ) encouraged us to approximate the data with a line $y=k$, where $k=$ mean $z P(A)$. The root-mean-square residual from $y=k$ was only .042. This small RMS error confirmed the result of the Newman-Keuls test: Performance is unaffected by time of arrival of the cue following the observation interval. The cue-before conditions were best described by a line in which $\mathrm{zP}(\mathrm{A})=.58 \mathrm{x}+.65\left(\mathrm{r}^{2}=.87\right)$. This confirms that as the interval between cue presentation and motion onset is increased, there is a linear increase in sensitivity.

\section{Discussion}

The first thing of note in Experiment 1 is the magnitude of the uncertainty effect: $P(A)$ ranged from 
.65 in the uncertainty condition to .86 in the certainty condition. These $P(A)$ values correspond to $d^{\prime} s$ of .51 to 1.53 , a range quite similar to that found previously using a two-alternative forced-choice procedure and different observers (Sekuler \& Ball, 1977). Therefore, under somewhat different conditions from those used previously, the major details of our earlier work on direction uncertainty have been replicated.

Next, consider the effect of the cue. Cuing an observer prior to the observation interval improved detection; when the observer was cued following his observation, however, the effect of uncertainty was not reduced. Although the differences were not statistically significant in most cases, performance was slightly worse when a cue followed the observation interval than when no cue was presented at all. This puzzled us. Upon questioning, two observers reported that occasionally, when the cue's direction surprised them (they thought they had seen a different direction of movement), they became less confident that a stimulus had been presented. If true, this would be an interesting state of affairs. Motion had been presented and actually perceived by the observer, but the observer would give responses indicating that no motion had been presented (" 4 ," " 5 ," or " 6 "). Such incorrect responses would reduce the observer's $\mathbf{z P}(\mathbf{A})$.

To check on the observers' reports, the three observers participated in one additional block of $\mathbf{8 0}$ trials in which the cue always followed the observation interval by $700 \mathrm{msec}$. On each trial, an observer first reported which direction he believed had been presented and then rated his confidence that moving dots had or had not been presented. For all three observers, when the direction response agreed with the actual direction presented, they were nearly always confident that moving dots had been presented. When the direction judgment did not agree with the actual direction presented, however, observers were much more likely to respond that no moving dots had been presented.

This information suggested that observers had been using cues delivered after the stimulus for confirmation. When a stimulus was presented and the cue confirmed what the observers believed they had seen, they responded that a stimulus had been presented (category response " 1 ," " 2 ," or " 3 ") $97 \%$ of the time. When a stimulus was presented, and the cue contradicted what the observers believed had been presented, confidence fell and observers used those same categories only $9 \%$ of the time. This resulted in slightly lower sensitivity, $\mathrm{zP}(\mathrm{A})$, when the cue followed the stimulus than when no cue was presented at all. The theoretical significance of this finding will be treated in detail in the General Discussion of this paper.

\section{EXPERIMENT 2}

Experiment 1 showed that sensitivity increased linearly with the interval by which the cue preceded the presentation of motion. This suggests that a certain amount of time is required following the presentation of a cue for the observer to become "set" for the appropriate stimulus. If no time were required, then even very short lead times would have sufficed to eliminate the effects of uncertainty. In fact, a lead time of $450-700 \mathrm{msec}$ between the cue and observation interval was needed, although shorter intervals reduced uncertainty considerably.

Experiment 2 investigated the informational requirements of an effective cue. In particular, it asked whether the cue must specify exactly which direction of movement would follow or whether cues that merely approximated the direction could also help. This information would be particularly important in the design of a sensory aid to reduce uncertainty. Feasible hardware devices would be more likely to provide rather general information to the human observer. This experiment attempted to determine just how precise a cue must be to benefit the observer.

\section{Method}

The second experiment used the same rating scale procedure as before to measure the detectability of moving dot patterns and the observer's criterion for reporting movement. The same three observers participated again and were tested at the same luminances as in Experiment 1.

Three types of sessions were run, each with 80 trials of the same type: certainty, uncertainty, or uncertainty plus cue. In all three conditions, movement was presented on half the trials, chosen at random. The CRT was blank, except for the veiling luminance, on the remaining trials. The certainty condition was the same as that in Experiment 1. The uncertainty condition, however, was changed. Movement could be in any of $\mathbf{3 6 0}$ different, evenly spaced directions. As before, the direction of movement was unpredictable from one trial to the next, and each direction had an equal probability of occurring.

The uncertainty-plus-cue condition contained seven different subconditions, defined by the size of the range represented by the cue (i.e., its unreliability). In each subcondition, the cue indicated, with varying degrees of reliability, the direction of motion that might follow. In all subconditions, the cue line preceded the observation interval by $700 \mathrm{msec}$. On each trial, the 360 possible cue orientations were all equally likely. We designate the seven subconditions as having unreliabilities of $360,300,240,180,120,60$, or $0 \mathrm{deg}$. On each trial, the computer first randomly selected a cue orientation and then randomly selected a direction of movement. If $x$ deg of cue unreliability were needed, the direction of movement was chosen from a uniform distribution centered about the cue's orientation and covering a range of directions $x$ deg wide.

To explain this further, consider three subconditions in detail. The 360-deg condition represents the least reliability, since the cue and target direction are completely uncorrelated. In this condition, regardless of the cue orientation, stimulus movement following the cue could be in any direction. In fact, the computer that ran the experiment first made a random choice of the cue orientation that would be presented and then made an independent random choice of the direction of movement. Both random choices were from a uniform distribution covering all $360 \mathrm{deg}$ in intervals of 1 deg. 
At the other extreme, in the 0-deg condition, the cue was perfectly reliable. On those trials during which motion was to be presented, the cue always pointed exactly in the direction of that motion. The 180-deg condition represents one intermediate amount of cue reliability. The computer first chose the direction of the cue at random and then chose the direction of movement from a truncated distribution covering $180 \mathrm{deg}$, centered about the direction indicated by the cue. Thus, from the observer's point of view, the motion could bracket the direction indicated by the cue by \pm 90 deg. An analogous procedure was used for other conditions of intermediate cue unreliability.

Recall that movement was presented on only half of the trials. On trials during which no movement would be presented, a randomly oriented cue was shown. Eighty trials with constant cue unreliability were run in a block, and, as before, observers rated their certainty that the moving dot pattern had been presented by responding with one of the six possible response buttons.

Observers were informed before each block of trials which condition or subcondition was to be presented so that they knew how wide a direction range to monitor. Each observer participated once in each condition or subcondition.

\section{Results}

$P(A)$ values were calculated and $z$ transformed as described earlier. In addition, the measure of criterion, B, was also calculated. Figure 2 shows detection performance for the three observers. The rightmost points in the figure show data for the certainty condition; the far left-hand points show results for the uncertainty condition. Between these two sets of data points are ones for the seven subconditions of cue reliability.

In general, as the cue became more reliable, sensitivity increased and the detrimental effects of uncertainty declined. The relationship between precision and detectability was well described by the equation $\mathrm{z} P(\mathrm{~A})=-.002 \mathrm{x}+1.02$, where $\mathrm{x}$ refers to the precision of the cue, in degrees. The quality of the fit $\left(r^{2}=.96\right)$ confirms the fact that detection improves linearly as the cue becomes more reliable. The $\mathrm{Y}$ inter-

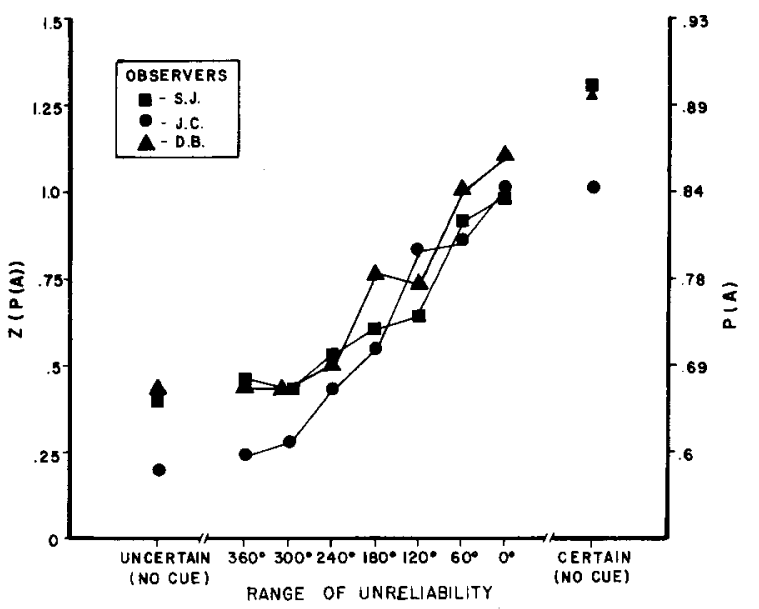

Figure 2. Sensitivity, $z P(A)$, for each condition and observer in Experiment 2. Rightmost points show certainty and far left-hand points show uncertainty. Sensitivity rises as cue reliablity increases. cept represents an average performance for the 0 -deg cue unreliability condition.

A one-way analysis of variance revealed a significant effect of cue unreliability $[F(8,16)=30.00, p<.01]$. A Newman-Keuls test produced two interesting results: first, that detection with 0 -deg cue unreliability was significantly worse than detection with certainty, and second, that no condition in which cue unreliability exceeded 180 deg was significantly better than the uncued uncertainty condition.

An analysis of variance showed no significant change in B across conditions, indicating an unchanged bias for or against saying a stimulus had been presented. Apparently, cue reliability alters $\mathrm{zP}(\mathrm{A})$ sensitivity but not the observers' criterion.

\section{Discussion}

Experiment 2 showed that a very precise cue presented well in advance of the target reduced the effect of uncertainty to a considerable degree. More importantly, the results show that a cue does not have to specify the direction of subsequent movement precisely in order to reduce the uncertainty effect. For example, a cue specifying the direction of movement to an accuracy of $60 \mathrm{deg}$ or better was sufficient to reduce the uncertainty effect by half.

Our terminology names each uncertainty range by the maximum possible unreliability for any condition. Although it is convenient, this terminology may be a bit misleading to those unfamiliar with it. For example, when cue unreliability was $120 \mathrm{deg}$, virtually all trials had cues that were much more precise. In this condition, the actual direction of motion could differ from the direction specified by the cue by as much as 60 deg. But the probability of this extreme discrepancy between cue and target direction was only $1.66 \%$. Likewise, the probability of the cue's being perfectly precise was $1.66 \%$. The mean absolute difference between the cue direction and direction of motion was $30 \mathrm{deg}$ in this condition. In other words, any single range of uncertainty encompasses many different degrees of discrepancy between cue and target direction. We will discuss the implications of this fact more fully in the General Discussion.

\section{EXPERIMENT 3}

Taken together, Experiments 1 and 2 show that sensitivity to a moving target improves linearly with either of two variables, precedence time and cue reliability. As mentioned previously, if hardware sensory aids are to help human observers faced with uncertainty about moving targets, the aids must deliver information in time to allow the observer to assimilate it and adjust his target search accordingly. Feasible sensory aids would not allow the observer much advance warning and, furthermore, are more likely to provide general information rather than specifying 
stimulus characteristics exactly. The results of Experiments 1 and 2 are encouraging with regard to the practicality of such a device, since they show that crude information could prove beneficial to our human observers and that observers do not require substantial lead times to adjust their sensory apparatus.

The information provided by Experiments 1 and 2 leaves several questions unanswered, however. Suppose, for example, that a potential sensory aid, by taking longer to analyze a target's characteristics, could provide somewhat more precise information to the observer. Would this extra delay be justified by improved observer performance? It might not be, if the increased analysis time delayed the delivery of the information until it was too late to help the observer. Experiment 3 assessed the tradeoff between lead time and reliability of a cue in order to determine what combinations of the two variables would result in optimum performance.

\section{Method}

Two observers participated in this experiment; neither had served in the preceding experiments. Preliminary measurements determined the appropriate incremental luminance at which to test each of these new observers. The luminance chosen for each observer provided approximately the same performance under conditions of certainty as had been obtained in the first two experiments.

Three different cue lead times were used, 700,400 , and 200 msec. Each was combined with seven different values of cue unreliability: $360,300,240,180,120,60$, and 0 deg.

The experiment used the same rating scale procedure as before. Each trial consisted of a warning signal, a 50 -msec presentation of the cue, a delay for one of the precedence times, and finally, the observation interval of $500 \mathrm{msec}$. Movement was presented on half of the trials. Before each session, observers were told which unreliability condition was to be presented. Each observer was tested once in each of the 21 combinations of lead time and cue unreliability. The order of conditions tested was randomly determined for each observer.

\section{Results}

$P(A)$ values were again computed, and their $z$ transforms were taken. Data from the two observers were virtually identical, and therefore the mean performance for the two observers is shown in Figure 3. $\mathbf{z P}(\mathrm{A})$ is shown as a function of cue unreliability for the three different lead times tested. For each precedence time, $\mathrm{zP}(\mathrm{A})$ declines monotonically with increasing cue unreliability. An analysis of variance revealed a significant effect of time of arrival of the cue $[\mathrm{F}(2,2)=219.31, \mathrm{p}<.01]$, as well as the unreliability of the cue $[F(6,6)=26.20, p<.01]$. The interaction between these two variables was also significant $[F(12,12)=12.94, p<.01]$. This interaction reflects the fact that performance was very bad at all preceding intervals as cue unreliability reached high values.

Using a Monte Carlo technique, we simulated 1,000 rating scale sessions like our own, but under conditions of no actual difference between signal and

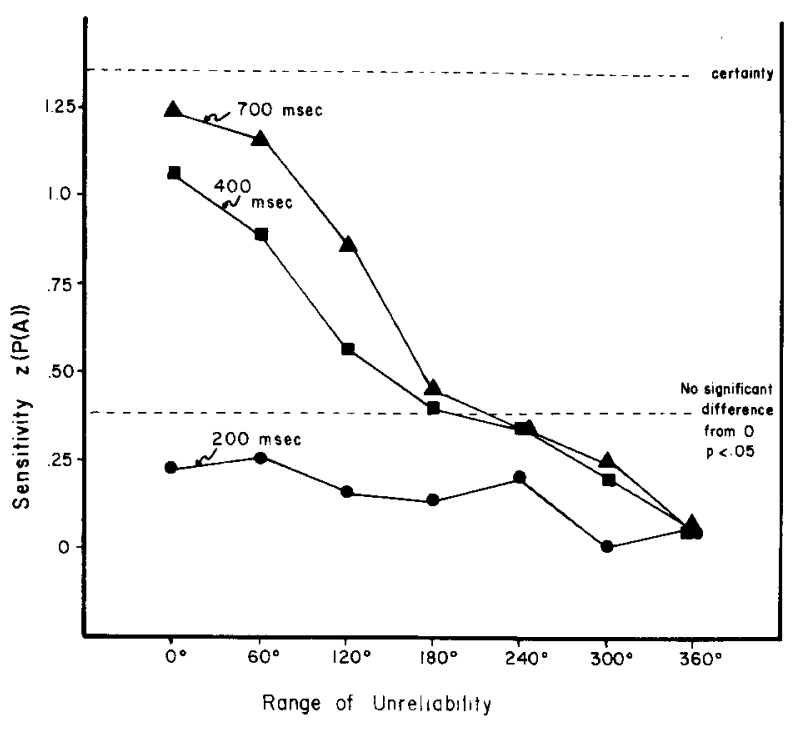

Figure 3. Senditivity, $\operatorname{xP}(A)$, as a function of cue unreliability for three different cue precedence times.

noise. The cumulated probabilities of $\mathrm{zP}(\mathrm{A}) \mathrm{s}$ showed that any $\mathrm{zP}(\mathrm{A})$ value less than or equal to .39 was not significantly different from chance $[\mathrm{zP}(\mathrm{A})=0.0]$, with $\mathrm{p}<.05$. Therefore, if cue unreliability was equal to or greater than $240 \mathrm{deg}$, performance was not significantly better than chance for all lead times. Moreover, none of the conditions with a cue lead time of 200 msec were significantly better than chance.

\section{Discussion}

The results described above confirm those of Experiments 1 and 2 using different observers. The data from this experiment can be further examined, however, since they represent sensitivity measures obtained with concurrent variation of reliability and lead time. We wished to determine first whether the effects of reliability and lead time were additive. The significant interaction in the analysis of variance suggested that they were not additive. But, of course, this interaction resulted in part from our dependent measure's finite lower bound (i.e., chance performance; see Figure 3). Consequently, we wished to test the additivity of precision and lead time in a way that would be only minimally affected by this lower bound.

With this aim in mind, we first determined how each variable decreased performance below that for the optimum combination of the two variables -0 deg unreliability and $700 \mathrm{msec}$ lead time. For example, combining $400 \mathrm{msec}$ precedence time with 0 -deg unreliability caused observer J.M.'s zP(A) to drop by .23 below that with the optimum combination. We took this as an estimate of the loss resulting from reducing lead time from 700 to $400 \mathrm{msec}$. The combination of $700 \mathrm{msec}$ and 60 deg of unreliability caused J.M.'s $z P(A)$ to drop by .18. We took this as an esti- 


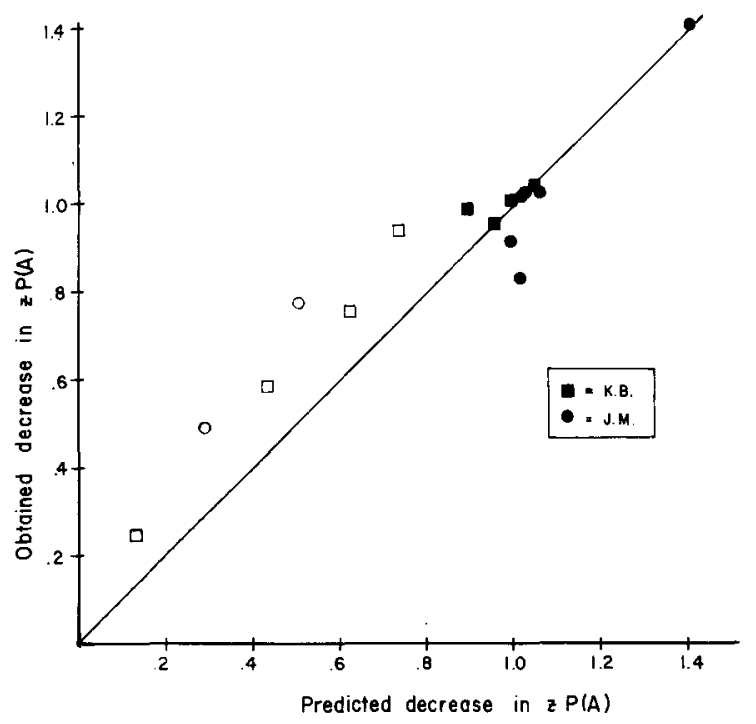

Figure 4. Predicted vs. actual losses in $\mathrm{zP}(\mathrm{A})$ are shown for two observers due to the combined effects of cue unreliability and cue precedence time. Actual losses exceed predicted losses from the Pythagorean theorem.

mate of the loss due to changing from perfect reliability to $60 \mathrm{deg}$ unreliability.

If losses due to reduced lead time and those due to cue unreliability were independent, the detectability loss caused by various combinations of the two variables should be predictable from the Pythagorean theorem (see Ball \& Sekuler, 1980). In particular, detection losses for any combination of lead time and cue unreliability should equal the square root of the two independent sources of losses squared and summed.

Predicted and actual losses are shown for the two observers in Figure 4. In general, losses obtained with combination of the two variables exceeded the losses predicted from the Pythagorean theorem. In Figure 4, solid points do not differ significantly from chance as determined by our Monte Carlo procedure. For the points remaining, losses are systematically greater than predicted. The effects of reduced precedence time and increased cue unreliability interact to produce a greater loss in detectability than expected from the sum of each separate effect.

\section{GENERAL DISCUSSION}

Because prior information about direction of movement is helpful to human observers, we tried to determine how this information should be delivered to the observers in order to reduce detection losses attributed to uncertainty. We first asked, in Experiment 1, how far in advance direction information should be available. Sensitivity increased linearly with the time of arrival of a direction cue. Practically speaking, then, up to some limit, the further in advance of the signal a cue is presented, the better performance should become. Note, however, that, at least under the conditions of Experiment 1, with only four possible directions, a cue delivered $450-700 \mathrm{msec}$ in advance of the stimulus was as effective as verbal instructions preceding the certainty condition by several minutes or more.

\section{Tradeoff Between Precedence Time and Cue Accuracy}

In Experiment 1, the effect of uncertainty on detection was reduced even when no time intervened between the offset of the cue and the onset of motion. We believe this occurred because the subject was able to adjust viewing strategy during the early part of the observation interval itself. Consistent with this conclusion, Ball and Sekuler (1981) examined detection with various lengths of observation intervals. With no cue, detection improved as duration of presentation grew from 100 to $600 \mathrm{msec}$. The rather long observation intervals $(500 \mathrm{msec})$ used in Experiment 1 probably allowed observers enough time first to incorporate the cue information and then to monitor the appropriate direction channel (albeit for a shorter period of time). We will return to this point again later.

Experiment 2 examined the effects of varying the precision of the cue. A cue did not need to specify the direction of subsequent movement precisely in order to reduce the uncertainty effect. This was encouraging with regard to the practicality of a sensory aid, but we still needed to know how the two variables, time and precision, interacted. Introducing Experiment 3, we asked about the appropriate lead-time/ precision tradeoff for possible sensory aids. For example, would it be more desirable for the aid to delay delivery of its information if that delay would allow the information, when delivered, to be more precise? The results of Experiment 3, precision and lead time, make it clear that a cuing device would provide more aid to an observer by using the time available to provide the maximum precision possible even at the expense of a considerably shortened preceding time.

\section{Time Needed to Switch Between}

Direction Mechanisms

Experiments on direction uncertainty have shown that observers are most sensitive to only a limited range of directions at any one moment in time. The observer can adjust the range monitored, but that change requires some finite period of time (Ball \& Sekuler, 1980). Using cues to indicate which direction of movement might follow appeared to be a good way to control the direction mechanism an observer would monitor. Presenting cues at various times relative to the observation interval allowed us to study how long it took an observer to switch to the appropriate direction following a cue. 
Experiment 1 showed that the time at which the cue was presented prior to the stimulus altered sensitivity. This information, however, was a general finding based on an entire block of 80 trials. In order to glean more information on the time required to switch attention from one direction to another, trialwise analysis of the data was performed. The logic behind this analysis was as follows: First, assume that it does require time to switch from one direction mechanism to another. If an observer's attention is centered on the appropriate direction at the end of each trial, then those trials for which the observer is cued to remain on the same direction should exhibit higher $P(A)$ values than those for which the observer is cued to switch. This should be especially noticeable on those trials in which there is very little time between the cue and observation interval.

Recall that in Experiment 1 four directions of motion were equally likely in a block of 80 trials (up, down, left, and right). As a result, we can define three types of trial pairs: those in which the cue direction was the same on both trials, those in which the cue direction changed by $90 \mathrm{deg}$ from one trial to the next, and those on which the cue direction changed by $180 \mathrm{deg}$. Responses for all three types of trial pairs were tabulated separately for each of the seven lead times $(0,50,100,200,300,450$, and $700 \mathrm{msec})$. As shown in Table 1, P(A) values were then calculated and $z$ was transformed for each type of trial pair. In each condition, both the trials that required no switching and those that required a 90-deg switch occurred approximately 100 times; trials that required a switch of $180 \mathrm{deg}$ occurred about half as often. As a result of separating the total number of trials into three types of trial pairs, variability was increased and the data in Table 1 appear somewhat ragged.

First, note that, for all three types of trial pairs, as the time interval between offset of the cue and the observation interval increases, $2 \mathrm{P}(\mathrm{A})$ also increases. This improvement was noted earlier in the main analysis of Experiment 1. But this analysis of trial pairs also shows another, new effect. At each lead time, pairs on which the cue direction remained the same produced higher performance than pairs on which a 90 - or 180 -deg switch was indicated. Furthermore,

Table 1

Values of zP(A) for Various Differences Between Directions on Trials $n$ and $n+\hat{i}$

\begin{tabular}{cccc}
\hline \multirow{2}{*}{$\begin{array}{c}\text { Precedence } \\
\text { (in Milliseconds) }\end{array}$} & \multicolumn{3}{c}{ Change From Trial n to $\mathrm{n}+1$} \\
\cline { 2 - 4 } & $0 \mathrm{deg}$ & $90 \mathrm{deg}$ & $180 \mathrm{deg}$ \\
\hline 0 & .61 & .39 & .13 \\
50 & .74 & .61 & .15 \\
100 & 1.04 & .64 & .33 \\
200 & 1.48 & .88 & .39 \\
300 & 1.23 & .67 & .39 \\
450 & 1.18 & .84 & .71 \\
700 & 1.48 & 1.18 & .92 \\
\hline
\end{tabular}

pairs on which a 90-deg switch was required produced higher $\mathrm{zP}(\mathrm{A})$ values than those on which a 180deg switch was indicated.

Table 1 reveals several other interesting findings. First, notice that, for trial pairs in which the cue remains the same, $z P(A)$ increases with lead time only up to $200 \mathrm{msec}$. For the four longest times $(200,300$, 450 , and $700 \mathrm{msec}$ ), performance remains the same. This indicates that a lead time of $200 \mathrm{msec}$ is sufficient for optimum performance when no switching is required of the observer. This interval $(200 \mathrm{msec})$ probably reflects the interval needed for the observer to incorporate the information provided by the cueto realize that no switching is required.

But what about the other two types of trials? When a 90-deg switch was required, $\mathrm{zP}(\mathrm{A})$ increased with lead time over the entire range; the same is true for pairs requiring a switch of $180 \mathrm{deg}$. This implies that a factor in addition to cue incorporation is operative. Since the only difference among the three types of trial pairs is the extent of a switch that was required, we must conclude that it requires time to make a switch. If it required $100-200 \mathrm{msec}$ to incorporate the cue information, plus an additional period of time to switch, then performance should be worse on trials in which a switch is required, since the effective observation interval would be shortened. This is, in fact, what we observed.

We may then ask how long it takes to make a switch from one direction to another. We may obtain a rough estimate of this time by determining the point at which performance becomes equivalent for the three different types of trial pairs. In looking at Table 1 , notice that performance on trial pairs in which a 90-deg switch was required resembled that for no switch and 0 -msec lead time in all of the $50-300-\mathrm{msec}$ conditions. For pairs in which a switch of $180 \mathrm{deg}$ was required, a preceding time of 450 msec was needed to reach a level of performance equivalent to that for no switch and 0 -msec lead time. Thus, it appears that somewhere between 50 and $300 \mathrm{msec}$ is required to make a switch of $90 \mathrm{deg}$ and as much as $450 \mathrm{msec}$ is required for a switch of $180 \mathrm{deg}$.

This analysis supports the idea that it takes time for an observer to prepare for a stimulus moving in a different direction. If the observer is prepared to detect an upward-moving target and suddenly must switch for a downward movement, for example, detectability will decline. This decline will be far less, however, if the lead time allows the observer to adjust the sensory apparatus without eating into the observation interval itself. When $700 \mathrm{msec}$ elapse between the offset of the cue and the onset of movement, we noted very little difference in sensitivity among the three different types of trial pairs, even when a 180-deg switch was required.

We pointed out earlier that the duration of the observation interval was important. Given a long ob- 
servation interval, observers in Experiment 1 were able to adjust their viewing strategy during the early part of the observation interval. This conclusion, added to the analysis of trial pairs, indicates that it is the total time (lead time plus observation interval), and not simply the interval between the cue offset and movement onset, which sets a limit on performance.

\section{Effects of Grossly Incorrect Cues}

Evidence presented so far has indicated that observers are sensitive to only a limited range of directions. When a fairly accurate cue is given $(0-120 \mathrm{deg}$ unreliability), performance improves because the range is appropriate for the stimulus. We wondered how a cue signaling a grossly incorrect direction of motion would affect sensitivity. To check this, we further analyzed data from Experiment 2 . We compared (1) trials on which the cue indicated a direction close to the actual direction presented with (2) trials on which the cue indicated a direction quite different from the actual direction presented. With 60 -deg reliability, trials were divided into those in which the direction of movement differed from the cue direction by $15 \mathrm{deg}$ or less and those that differed by more than $15 \mathrm{deg}$. For example, if the cue indicated a direction of $90 \mathrm{deg}$, trials in which movement direction fell in the range from 75 to 105 deg were separated from trials in which movement direction was 105 to $120 \mathrm{deg}$ or 60 to $75 \mathrm{deg}$. With 120 -deg reliability, trials in which the cue direction and subsequent direction of movement differed by $30 \mathrm{deg}$ or less (half of the 120-deg range) were separated from those that differed by more than $30 \mathrm{deg}$ on either side. After making this division for each condition, $\mathrm{zP}(\mathrm{A})$ values were again calculated. An analysis of variance revealed a significant difference between the trials on which cue direction and motion direction differed by half or less of the total range and those on which the difference was more than half the total range $[F(1,2)$ $=33.83, \mathrm{p}<.05]$.

These results have several important implications. First of all, they remind us of the heterogeneity of the data from each unreliability condition in Experiment 2. Performance levels we previously presented for Experiment 2 were means of all the trials in each range, and, as indicated earlier, most trials were more precise than the names of the unreliability ranges imply.

Second, this analysis allows us to determine the effect of a grossly incorrect cue. Figure 5 represents graphically the effect on performance as the difference between the cue direction and actual direction of movement grows. Notice that, for large differences between these two directions (90 to $180 \mathrm{deg}$ ), performance is depressed below the level achieved with no cue at all (indicated by the dashed line). Thus, an incorrect cue that centers an observer's sensitivity on a direction differing from the actual direction by

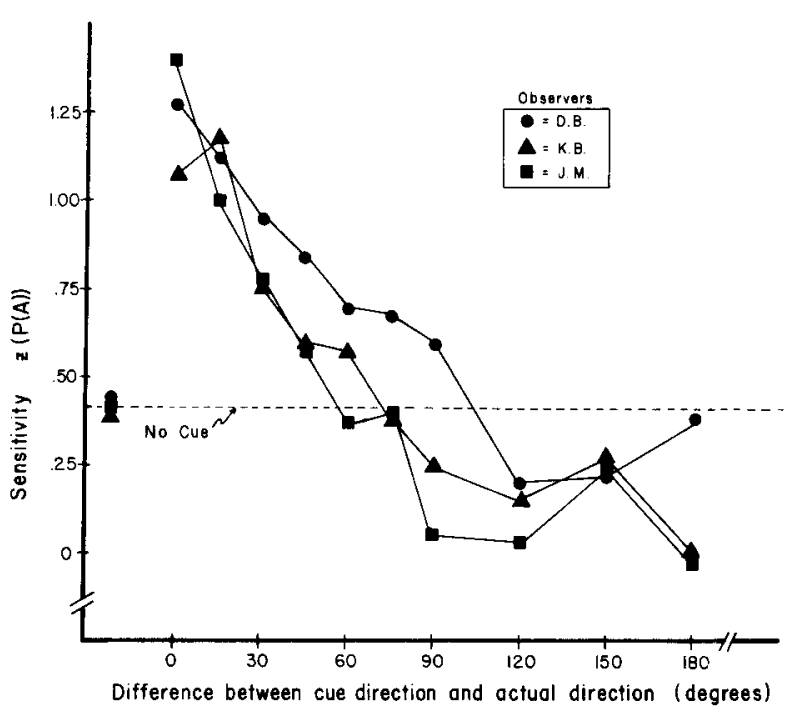

Figure 5. Sensitivity, $z P(A)$, as a function of the difference between cue direction and movement direction. For large differences between these two directions, performance is depressed below the level achieved with no cue (dashed line).

90 deg or more results in a cost in performance: It is worse than no cue at all. We believe this stems from the fact that the incorrect cue consistently diverts the observer's sensitivity to a range inappropriate for detecting the stimulus. Probability dictates that, with uncertainty and no cue whatever, there will be trials on which the observer's sensitivity is centered correctly, or very nearly so. There will also be trials on which sensitivity is inappropriately centered. The details of the mixture depends upon chance to some extent, but the result is clear: an average performance surpassing that produced with a consistently inappropriate centering of sensitivity. This kind of consistent error would arise if the cue always misled the observer by a wide margin.

\section{Breadth of Tuning in Direction Mechanisms}

Let us now consider how these experiments bear upon the visual mechanisms enabling us to see moving targets. First of all, these mechanisms may be described as "directionally selective" filters, attenuating some input signals-stimuli moving in one direction or another-more than others. We already know several characteristics of these filters. Previous experiments have shown them to be symmetric, monotonic, and rather broadly tuned in comparison with orientation-selective mechanisms (Ball \& Sekuler, 1979, 1980; Levinson \& Sekuler, 1980). Furthermore, sensitivity to moving targets is independent of their direction of motion. This means that all directionselective mechanisms are equally sensitive at their peak or center direction (Ball \& Sekuler, 1980; Levinson \& Sekuler, 1980; Marshak, Note 1).

The results of the analysis just described allow us to estimate the breadth of tuning of the underlying 
direction-selective mechanisms. Lines were fit for each observer for all the conditions representing differences between 0 and $90 \mathrm{deg}$ to determine the difference at which performance equaled that of the nocue condition. This point of equality indicates the cutoff between a cue that is of benefit to the observer and a cue that is harmful to performance. Performance became equivalent to the no-cue condition for the three observers at differences of 55, 74, and $97 \mathrm{deg}-\mathrm{a}$ mean of $75 \mathrm{deg}$. Since the data were blocked in 15-deg sections to provide enough trials to determine $P(A)$, the precision of our estimates is limited. Nevertheless, the results are remarkably consistent with past tuning estimates (Ball \& Sekuler, 1979, 1980; Levinson \& Sekuler, 1980).

\section{Individual Differences}

It appears from our experiments that uncertainty may prove more harmful to some observers than to others. For example, lead time appears to have played a more significant role for the two observers in Experiment 3 than it did for the three observers in Experiment 1. In particular, performance is far lower for the 200-msec condition in Experiment 3 than it was in Experiment 1, even though performance in the certainty condition was equivalent for all observers. These types of individual differences have been observed previously and may reflect the different detection strategies employed by the observers (Ball \& Sekuler, 1981).

\section{Conclusions}

The three experiments presented here have shown that rapid deployment of sensory resources is possible. These results are encouraging with regard to the design of a sensory aid and the benefits such a device would provide in reducing uncertainty. One final comment with regard to such a device: Recall that, in Experiment 1, observers who received cues following their observation were sometimes surprised by the direction indicated by the cue. This implies that an observer's ability to identify a direction of motion that has been presented may not always coincide with ability to detect the presence of movement. Preliminary results on this question have shown some rather striking discrepancies between detection performance-simply seeing the motion-and the ability to assess direction of motion (Ball, Machamer, \& Sekuler, Note 2). In particular, the relationship between identifiability of direction and detection of its presence varied as a function of the range of possible directions. This distinction between detection and identification may have important implications in the design of a hardware sensory aid. Since we cannot guarantee good identifiability simply by arranging conditions to provide good detectability, this distinction may become important, depending upon the uses for which the device is needed.

\section{REFERENCE NOTES}

1. Marshak, W. Personal communication, 1980.

2. Ball, K., Machamer, J., \& Sekuler, R. Detection and identification of motion with direction uncertainty. Paper presented at the meeting of the Optical Society of America, Chicago, 1980.

\section{REFERENCES}

Andrews, D. P. Perception of contours in the central fovea. Nature, 1965, 205, 1218-1220.

Appelle, S. Perception and discrimination as a function of stimulus orientation: The "oblique effect" in man and animals. Psychological Bulletin, 1972, 78, 266-278.

Ball, K., \& SE kuler, R. Masking of motion by broadband and filtered directional noise. Perception \& Psychophysics, 1979, 26, 206-214

Ball, K., \& Sexuler, R. Models of stimulus uncertainty in motion perception. Psychological Review, 1980, 87, 435-469.

Ball, K., \& Sekuler, R. Adaptive processing of visual motion. Journal of Experimental Psychology: Human Perception and Performance, 1981, 7, 780-794.

Cohn, T. E., \& LAsley, D. J. Detectability of a luminance increment: Effect of spatial uncertainty. Journal of the Optical Society of America, 1974, 64, 1715-1719.

Greenhouse, D. S., \& Cohn, T. E. Effect of chromatic uncertainty on detectability of a visual stimulus. Journal of the Optical Society of America, 1978, 68, 266-267.

Johnson, D. M., \& HAFTER, E. R. Uncertain-frequency detection: Cuing and condition of observation. Perception \& Psychophysics, 1980, 28, 143-149.

Levinson, E., \& Sekuler, R. A two-dimensional analysis of direction-specific adaptation. Vision Research, 1980, 20, 103-107.

McNicou, D. A primer of signal detection theory. London: Allen \&Unwin, 1972

Seruler, R., \& Ball, K. Mental set alters visibility of moving targets. Science, 1977, 198, 60-62.

(Manuscript received December 1, 1980; revision accepted for publication May 18, 1981.) 